\title{
Deuxième intervention du Conseil fédéral dans le TARMED
}

Thomas Kessler ${ }^{a}$, Patrick Müller

a Expert, division Médecine et tarifs ambulatoires, FMH; b chef de la division Médecine et tarifs ambulatoires, FMH

Le Conseil fédéral fait à nouveau usage de la compétence subsidiaire que lui confère la loi en intervenant pour la deuxième fois dans la structure tarifaire nationale TARMED. Cette deuxième intervention prévoit de nombreux et importants changements dans le TARMED et concerne l'ensemble des médecins. L'ordonnance définitive avec tous les détails de l'intervention a désormais été publiée et entrera en vigueur au $1^{\text {er }}$ janvier 2018. Les principaux points sont présentés dans l'article qui suit.

De nombreuses organisations médicales et quelques médecins particuliers ont profité des trois mois de consultation sur la deuxième intervention subsidiaire du Conseil fédéral dans le TARMED pour transmettre leur prise de position à l'Office fédéral de la santé publique (OFSP). Le 20 juin 2017, la FMH a tenu une conférence de presse à Berne et présenté sa prise de position rédigée en étroite collaboration avec de nombreuses organisations faitières et spécialisées. Un extrait a été publié dans le Bulletin des médecins suisses 28/29 du 12 juillet 2017 et peut être consulté sur le site internet de la FMH.

Le département Médecine et tarifs ambulatoires de la FMH a procédé à des analyses et simulations détaillées des mesures de cette deuxième intervention avec les sociétés de discipline médicale et les sociétés cantonales de médecine. Ces analyses montrent que les vastes mesures d'économies prévues auront un impact considérable sur la prise en charge ambulatoire (restrictions de prestations sous la forme de «limitations»), mettant en péril les objectifs de la stratégie "Santé2020» du Conseil fédéral. La pratique libérale et les services ambulatoires des hôpitaux seront eux aussi en partie fortement touchés sur le plan financier.

Les mesures de l'ordonnance du Conseil fédéral, désormais définitive, affaibliront considérablement le secteur ambulatoire et n'inciteront aucunement à fournir les prestations dans ce secteur, pourtant nettement moins cher et plus efficace, menaçant ainsi la prise en charge ambulatoire de la population rurale déjà problématique aujourd'hui.

\section{Impact sur le domaine LAA}

L'ordonnance du Conseil fédéral porte exclusivement sur l'assurance-maladie sociale LAMal. Le 18 septembre 2017, la CTM a décidé que la version actuelle du TARMED (01.08.00_BR) restera en vigueur jusqu'au 31 mars 2018 sans aucun changement, et que la nouvelle version 01.09.00_BR n'entrera en vigueur qu'au $1^{\mathrm{er}}$ avril 2018, avec des positions LAA supplémentaires et une revalorisation de certaines prestations (p. ex. expertises). Aucun détail n'est encore connu, mais cela signifie d'ores et déjà que deux structures tarifaires ambulatoires TARMED différentes seront appliquées en parallèle au moins pendant le premier trimestre 2018 (différenciation selon la loi appliquée LAMal ou LAA). Cette coexistence de deux structures tarifaires entraînera des difficultés d'application considérables.

\section{Deuxième intervention du Conseil fédéral dans la structure tarifaire TARMED au $1^{\text {er }}$ janvier 2018: les mesures}

Nous vous présentons ci-dessous les principales mesures de la deuxième intervention tarifaire du Conseil

\section{Important!}

N'oubliez pas de mettre à jour votre logiciel de facturation au cabinet avec la nouvelle structure tarifaire TARMED, version 1.09.00_BR, afin de pouvoir continuer de facturer vos prestations dans le cadre de l'assurance-maladie sociale LAMal à partir du $1^{\text {er }}$ janvier 2018. Votre fournisseur de logiciel vous épaulera volontiers. 
fédéral dans la structure tarifaire TARMED à partir du $1^{\text {er }}$ janvier 2018:

\section{Facteur de valeur intrinsèque uniforme pour toutes les prestations}

Les «valeurs intrinsèques quantitatives» sont uniformisées. Elles se voient toutes (FMHO5 à FMH12) attribuer un facteur de valeur intrinsèque uniforme de 0,985 . Si les points tarifaires de la prestation médicale (PM) des positions avec la valeur intrinsèque quantitative FMH5 (p. ex. 00.0010 Consultation, première période de $5 \mathrm{~min}$ [consultation de base]) augmentent (facteur initial $=0,905)$, ceux de toutes les positions tarifaires avec une "valeur intrinsèque quantitative» plus élevée (FMHO6 à FMH12) diminuent au facteur 0,985 . La prestation médicale (PM) du médecin praticien est abaissée au facteur 0,93 (base des nouveaux points tarifaires PM).

\section{Augmentation de la productivité médicale pour les unités fonctionnelles des salles d'opération}

La productivité des unités fonctionnelles des salles d'opération (OPI à OPIII) est revue à la hausse. La productivité des unités fonctionnelles OP I augmente de $45 \%$ à $55 \%$ (OPII de 50\% à $60 \%$ et OPIII de $55 \%$ à $65 \%$ ), réduisant dans le même temps les taux de coûts des prestations techniques (PT). En plus du revenu de référence, du temps de travail annuel et de la "valeur intrinsèque quantitative», la productivité médicale est un paramètre décisif pour le calcul du nombre de points tarifaires de la prestation médicale.

\section{Baisse des taux de coûts dans certaines unités fonctionnelles}

Le taux de coûts des unités fonctionnelles avec des coûts d'investissement pour installation, appareils et instruments supérieurs à CHF 750000 baisse de $10 \%$. Cette mesure concerne 25 unités fonctionnelles dont la salle d'opération de cabinet médical et la salle d'opération OP I. Les unités fonctionnelles UBR (salle de traitement et d'examen), dont par exemple UBR médecin de premier recours, UBR chirurgie et chirurgie pédiatrique, UBR ophtalmologie, UBR ORL, UBR urologie, UBR dermatologie et UBR angiologie, ainsi que l'unité fonctionnelle Endoscopie gastroentérologique, grande, ne sont pas concernées par cette mesure.

\section{Adaptation du minutage pour les prestations au sens restreint lors d'examens CT et IRM}

La prestation au sens restreint est supprimée sur les positions tarifaires pour les examens CT et IRM. Désormais, il existe une position tarifaire séparée pour les

\section{Visualisez l'impact de l'intervention}

\section{tarifaire sur vos propres prestations}

Quel sera l'impact de l'intervention tarifaire du Conseil fédéral et de ses mesures sur mon cabinet? Dois-je m'attendre à subir des pertes financières? Ce sont là quelques exemples des questions qui parviennent chaque jour au département Médecine et tarifs ambulatoires de la FMH.

L'outil Volumis Online permet à tous les membres de la FMH de répondre à ces questions et de visualiser l'impact de l'intervention tarifaire sur leurs propres prestations.

Vous trouverez de plus amples informations sur myFMH et sur notre site internet: www.fmh.ch $\rightarrow$ Tarifs ambulatoires $\rightarrow$ TARMED Tarif $\rightarrow$ Modifications du TARMED à partir du $1^{\text {er }}$ janvier 2018

prestations au sens restreint au cas où le spécialiste en radiologie doit être présent pour un certain temps lors d'un examen CT ou IRM. Cette position tarifaire est tarifée en tant que prestation à l'acte avec une prestation au sens restreint de 5 minutes. De plus, le minutage pour l'établissement de rapports lors de CT ou d'IRM est tarifé de manière uniforme, respectivement à 20 et 25 minutes.

\section{Diminution du minutage pour des positions tarifaires choisies}

Le minutage de la prestation au sens restreint respectivement du temps d'occupation du local est abaissé pour les prestations suivantes: opération de la cataracte, biopsie du corps vitré (et/ou injection intravitréale), ECG d'effort et de Holter, coloscopie et radiothérapie stéréotaxique.

\section{Modification du type de prestation:}

l'uexamen par le spécialiste» passe d'une prestation à l'acte à une prestation au temps

Les positions tarifaires «Examen par le spécialiste....» sont converties de prestations à l'acte en prestations au temps. Ces positions tarifaires seront facturées à l'avenir par période de 5 minutes. Cette mesure concerne 10 positions tarifaires dont 00.0410 Petit examen par le spécialiste de premier recours $(15 \mathrm{~min})$ remplacée par la position 00.0415 Petit examen par le spécialiste de premier recours, par période de $5 \mathrm{~min}$, et 00.0420 Examen complet par le spécialiste de premier recours (25 min) remplacée par 00.0425 Examen complet par le spécialiste de premier recours, par période de $5 \mathrm{~min}$.

\section{Application des limitations de facturation aussi aux spécialistes qui facturent par voie électronique}

La mention «Cette restriction ne concerne pas les spécialistes facturant par voie électronique» est supprimée (sans aucun remplacement) sur 20 positions tari- 
faires. Cette mesure concerne par exemple $00.0020+$ Consultation, par période de 5 min en plus (supplément de consultation), $00.0070+$ Visite, par période de 5 min en plus (supplément de visite) ou 00.0120 + Consultation téléphonique par le spécialiste, par période de $5 \mathrm{~min}$ en plus. Les limitations à $20 \mathrm{~min}$ sont doublées à 40 min pour les enfants $<6$ ans et les adultes $>75$ ans. Pour les patients présentant des besoins en soins plus importants, les limitations peuvent également être doublées. Pour les enfants $<6$ ans et les adultes $>75$ ans ainsi que les patients présentant des besoins en soins plus importants, des positions séparées sont désormais introduites. Les raisons justifiant les besoins plus importants en soins d'un patient doivent être inscrites dans le dossier du patient et dûment motivées auprès de l'assureur.

\section{Différenciation pour les "prestations en l'absence du patient"}

La position tarifaire 00.0140 Prestation médicale en l'absence du patient (y compris étude de dossier), par période de $5 \mathrm{~min}$, est supprimée en faveur des nouvelles positions suivantes:

- 00.0141 Etude de dossier, en l'absence du patient, par période de $1 \mathrm{~min}$

- 00.0142 Obtention d'informations auprès de tiers, en l'absence du patient, par période de $1 \mathrm{~min}$

- 00.0143 Renseignements donnés aux proches ou à d'autres personnes de référence du patient, en l'absence du patient, par période de $1 \mathrm{~min}$

- 00.0144 Discussions avec des thérapeutes et des soignants, en l'absence du patient, par période de $1 \mathrm{~min}$

- O0.0145 Envoi chez un médecin consultant, en l'absence du patient, par période de $1 \mathrm{~min}$

- 00.0146 Etablissement d'ordonnances ou prescriptions en dehors des consultations, visites et consultations téléphoniques, en l'absence du patient, par période de $1 \mathrm{~min}$

- 00.0147 Prestations diagnostiques d'instituts de pathologie, en l'absence du patient, par période de $1 \mathrm{~min}$

- 00.0148 Tumorboard en l'absence du patient, pour les personnes au-dessus de 6 ans et de moins de 75 ans, par période de $1 \mathrm{~min}$

La limitation actuelle de la position tarifaire est divisée par deux. Les limitations pour les enfants $<6$ ans et les adultes $>75$ ans ne sont pas divisées par deux. Pour les patients présentant des besoins en soins plus importants, les limitations peuvent également être doublées. Pour les enfants $<6$ ans et les adultes $>75$ ans ainsi que les patients présentant des besoins en soins plus importants, des positions séparées sont désor- mais introduites. Les raisons justifiant les besoins plus importants en soins d'un patient doivent être inscrites dans le dossier du patient et dûment motivées auprès de l'assureur.

En plus des prestations déjà inscrites dans l'interprétation de la position 00.0140, une nouvelle position «Tumorboards en l'absence du patient» est créée pour la participation aux tumorboards.

La même différenciation s'applique aux positions tarifaires 02.0070 Prestation médicale en l'absence du patient (y compris l'étude du dossier), par le spécialiste en psychiatrie, par période de $5 \mathrm{~min}$, et 02.0160 Prestation en l'absence du patient, par le psychologue ou psychothérapeute traitant, par période de $5 \mathrm{~min}$.

\section{Suppléments pour l'indemnité forfaitaire de dérangement en cas d'urgence}

L'interprétation des positions tarifaires comprenant la désignation «Indemnité forfaitaire de dérangement en cas d'urgence» est précisée. Les critères d'urgence durant la journée sont mieux délimités de façon à clarifier les cas dans lesquels l'indemnité forfaitaire peut être facturée. Les indemnités forfaitaires de dérangement en cas d'urgence peuvent être facturées aussi bien par les médecins que par les institutions de soins ambulatoires au sens de l'art. 36a LAMal.

Un point supplémentaire vient compléter les critères d'urgence utilisés jusqu'à présent pour l'indemnité forfaitaire de dérangement en cas d'urgence A (lu-ve 7-19, sa 7-12): si en cas de contact direct entre le patient et le médecin, un trouble des fonctions vitales est présent, à craindre ou ne peut pas être exclu, on se trouve désormais dans un cas d'urgence. Cette position peut aussi être facturée en cas de maladie aiguë, de traumatisme ou d'intoxication qui provoque ou qui peut provoquer la lésion d'un ou plusieurs organes. Dans le domaine de la psychiatrie, on partira du principe qu'il s'agit d'une urgence en cas d'état d'excitation, de mise en danger de soi-même ou d'autrui, de troubles de la conscience ou d'états stuporeux catatoniques. Lorsqu'il n'y a pas contact direct entre médecin et patient (p. ex. téléphone), les critères actuels restent applicables.

Pour les indemnités forfaitaires de dérangement en cas d'urgence B et C (lu-di 19-22, sa 12-19, di 7-19 et lu-di 22-7), les critères d'urgence sont complétés de sorte que, lors d'un contact direct entre médecin et patient, le traitement immédiat est jugé médicalement nécessaire par le spécialiste. Lorsqu'il n'y a pas contact direct entre médecin et patient (p. ex. téléphone), les critères actuels restent applicables, c.à.d. que le traitement immédiat est médicalement nécessaire et/ou jugé manifestement nécessaire par le patient, ses proches ou des tiers. Enfin, la fenêtre temporelle pour les 
indemnités forfaitaires de dérangement en cas de consultation ou visite pressante F/Visites en dehors des heures régulières de consultation (lu-ve 19-22, sa 12-19, et di 7-19) est étendue aussi à samedi matin (7-12).

\section{Adaptations de l'interprétation en cas de ponction et prise de sang par du personnel non médical (00.0715 et 00.0716)}

Dans l'interprétation des positions tarifaires 00.0715 Ponction veineuse pour prise de sang, toute localisation, par du personnel non médical et 00.0716 Prise de sang capillaire, toute localisation, par du personnel non médical, la limitation selon laquelle celles-ci ne peuvent être pratiquées que par le personnel du laboratoire de cabinet médical dans le cadre du diagnostic en présence du patient est supprimée. La prise de sang par du personnel non médical peut aussi être facturée si l'analyse s'effectue dans un laboratoire extérieur et pas au cabinet médical.

\section{Instruction du patient par le spécialiste} La position tarifaire 00.0610 Instruction du patient par le spécialiste pour lui apprendre à effectuer luimême des mesures ou des soins, par période de $5 \mathrm{~min}$, est désormais tarifée dans l'unité fonctionnelle salle de consultation et plus dans l'unité fonctionnelle UBR (salle d'examen et de traitement) médecin de premier recours.

FMH / Division Médecine et tarifs ambulatoires Baslerstrasse 47 CH-4600 Olten Tél. 0313591230 Fax 0313591238 tarife.ambulant[at]fmh.ch

\section{Suppression du supplément de $10 \%$ sur le matériel à usage courant et sur les implants} Le supplément de $10 \%$ sur le prix coûtant du matériel à usage courant et sur les implants est supprimé conformément à l'interprétation générale IG-20 Matériel à usage courant et implants.

\section{Modifications supplémentaires}

La position tarifaire 00.2205 Rapport médical sur formulaire, jusqu'ici facturée à l'acte, est désormais facturée au temps avec une limitation (max. 2 fois par séance) et sous un nouveau numéro (00.2206).

Une nouvelle position tarifaire 02.0015 + Indemnité de déplacement pour le spécialiste en psychiatrie et psychothérapie, par période de 5 min est introduite. La position est tarifée comme les diagnostic et thérapie psychiatriques.

Enfin, la tarification de la phytothérapie est identique à celle des autres formes de médecine complémentaire.

\section{La FMH vous aide à préparer la mise en œuvre}

Vous trouverez de plus amples informations sur la deuxième intervention du Conseil fédéral dans la structure tarifaire TARMED au $1^{\text {er }}$ janvier 2018 sur le site internet de la FMH à l'adresse www.fmh.ch $\rightarrow$ TARMED $\rightarrow$ Modifications du TARMED à partir du $1^{\text {er }}$ janvier 2018. Vous y trouverez également l'ordonnance et les documents afférents, un navigateur tarifaire avec mode d'emploi et banque de données, ainsi que diverses autres publications à ce sujet dont une foire aux questions (FAQ) et une fiche d'information. La FMH reste convaincue que seule une révision globale et complète du TARMED aux côtés de toutes les organisations médicales et de tous les partenaires tarifaires pourra redonner son caractère approprié et son économicité à la structure tarifaire. C'est dans ce but qu'elle poursuit activement le projet TARCO. 